\title{
Association between smoking cessation and post-hospitalization healthcare costs: a matched cohort analysis
}

\author{
Margaret B. Nolan', Bijan J. Borah², James P. Moriarty² and David O. Warner ${ }^{1 *}$ (D)
}

\begin{abstract}
Background: The potential economic benefit in terms of reduced healthcare costs when patients quit smoking after hospital discharge has not been directly measured. The aim of this study was to compare the costs for hospital admission and six-month follow-up for a cohort of patients who self-reported abstinence from cigarettes at 6 months after hospital discharge and a matched group of patients who reported continued smoking.

Materials and methods: This was a secondary analysis of a recent population-based clinical trial cohort (ClinicalTrials.gov ID: NCT01575145), with cohort membership determined by self-reported 7 day point prevalence abstinence at 6 months after the index hospital discharge. Participants were admitted to Mayo Clinic Hospital, Rochester, MN, between May 5, 2012 and August 10, 2014 for any indication and lived in the areas covered by postal codes included in Olmsted County, MN. Propensity score matching was used to control for differences between groups other than smoking status, and any residual imbalance was adjusted through generalized linear model with gamma distribution for cost and log-link transformation.

Results: Of 600 patients enrolled in the clinical trial, 144 could be contacted and self-reported 7 day point prevalence abstinence at 6 months after hospital discharge. Of these patients, 99 were successfully matched for this analysis. The cost for the index hospitalization was significantly greater in patients who abstained compared to those that did not abstain (mean difference of $\$ 3042$, higher for abstainers, $95 \% \mathrm{Cl} \$ 170$ to $\$ 5913, P=0.038$ ). However, there was no difference between mean 6-month follow-up costs, number of inpatient hospitalizations, or number of emergency room visits for abstainers versus non-abstainers.

Conclusion: There was no evidence to support the hypothesis that abstinence at 6 months after hospital discharge is associated with a decrease in health care costs or utilization over the first 6 months after hospital discharge.
\end{abstract}

Keywords: Smoking, Healthcare costs, Smoking cessation, Hospital costs

\section{Background}

An estimated 5 million patients who smoke require admission to US hospitals annually [1]. Hospitalization provides an opportunity to intervene in smoking behavior, and multiple studies have shown that interventions combining counseling with post-discharge follow-up can be efficacious [2], although the dissemination and implementation of such interventions has proved challenging in real-world practice. One of the potential benefits of

\footnotetext{
* Correspondence: Warner.david@mayo.edu

'Department of Anesthesiology and Perioperative Medicine, Mayo Clinic, 200 First Street SW, Rochester, MN 55905, USA

Full list of author information is available at the end of the article
}

successful interventions could be a reduction in the costs of medical care after hospital discharge. For example, improvement of smoking-related illness could result in lower readmission rates. Most of the economic analyses regarding the potential impact of smoking cessation on costs rely upon estimates of the costs of smoking-related diseases, and assumptions of how cessation will affect the prevalence of such diseases, rather than direct measurements of costs $[3,4]$. In a prior analysis, we demonstrated that smokers undergoing elective surgery incurred the same costs for the index hospitalization compared with never-smokers undergoing the same procedure, but experienced greater costs in the first year

(c) The Author(s). 2019 Open Access This article is distributed under the terms of the Creative Commons Attribution 4.0 International License (http://creativecommons.org/licenses/by/4.0/), which permits unrestricted use, distribution, and reproduction in any medium, provided you give appropriate credit to the original author(s) and the source, provide a link to the Creative Commons license, and indicate if changes were made. The Creative Commons Public Domain Dedication waiver (http://creativecommons.org/publicdomain/zero/1.0/) applies to the data made available in this article, unless otherwise stated. 
after discharge [5]. However, this analysis included only surgical patients and did not include any information regarding smoking status post-discharge, so the potential influence of postoperative abstinence on costs in patients who were smoking at the time of admission could not be measured.

We recently published a controlled trial (ClinicalTrials. gov ID: NCT01575145) in which 600 residents of Olmsted County, MN who were hospitalized at Mayo Clinic Rochester hospitals for any indication were randomized to receive an intervention aimed at facilitating telephone counseling ("quitline") utilization or a standard brief smoking intervention to explore the efficacy of using telephone quitlines in hospitalized patients to achieve smoking cessation [6]. The quitline facilitation intervention did not change self-reported abstinence rates 6 months after hospital discharge compared with a standard brief intervention ( $24 \%$ in both groups). While there is thus no rationale for analyzing healthcare costs according to treatment assignment, these data do provide the opportunity to explore the association of post-discharge abstinence with healthcare costs in a geographically-defined population across all admission indications.

This secondary analysis of a population-based study compares healthcare costs from hospital admission to 6 months after hospital discharge for those patients in the clinical trial who self-reported abstinence at 6 months and a matched group of trial patients who reported continued smoking. We hypothesized that costs in the 6 month period after discharge would be less in those patients who self-reported abstinence.

\section{Methods}

Both Mayo Clinic and Olmsted Medical Center Institutional Review Boards approved this study.

\section{Study population}

Patients for this analysis were sampled from participants in the randomized clinical trial with whom contact was made at 6 months after hospital discharge to ascertain smoking status. Patients eligible for the trial included those who lived in the areas covered by ZIP codes within Olmsted County, MN (55901-55906, which included all residents of Olmsted County and some who resided just outside of the county), were hospitalized in Mayo Clinic, Rochester between May 5, 2012 and August 10, 2014 for any admission indication, and reported current smoking at the time of admission. All indications were included to examine the association of abstinence with posthospitalization costs within all members of a geographically-defined population. In conformity with Minnesota statutes, patients that did not authorize the use of their medical record for research were excluded from the analysis.

\section{Data sources}

Data for the study patients including demographic and clinical characteristics were collected from electronic databases. Admission diagnosis was categorized using Clinical Classification Software (CCS) categories based on the primary diagnosis [7]. The Rochester Epidemiology Project Cost Data Warehouse was used to capture standardized cost data for the services that the patients received [8]. This includes costs for services provided by the two healthcare systems serving local residents, the Mayo Clinic and Olmsted Medical Center. In essence, the standardized cost data were estimated by adopting a hybrid approach in which Medicare reimbursement rates were assigned to all professional billed services, while the appropriate cost-to-charge ratios from the Medicare Cost Report were multiplied by the charges for all hospital-billed services [9].

\section{Statistical analysis}

Propensity score matching was conducted to select the non-abstinent cohort based on baseline characteristics including age, gender, and clinical indication based on CCS. The propensity score model used logistic regression with a one-to-one matching approach without replacement. Patients were matched using the nearest neighbor method with a 0.001 caliper. Common support was implemented. Pre- and post-match balance was assessed using the t-test for continuous covariates, and chi-square tests for categorical covariates. Any residual imbalance following propensity score matching was adjusted through generalized linear modeling (GLM). Both index hospitalization and 6-month post-discharge costs were reported for the abstinent and non-abstinent cohorts, along with the mean difference in costs. In an additional analysis, 6-month post-discharge costs were adjusted for index hospitalization costs. All GLM models for cost analyses assumed a gamma distribution for cost and a log-link to account for the skewed nature of the cost costs distribution [10]. Predicted differences in costs between the two groups were estimated. Statistical difference was determined using the $95 \%$ confidence intervals (CI) of the difference of the mean predicted costs. Assessment was also performed regarding whether the abstinent and non-abstinent patients differed in terms of their rate of readmission or emergency room (ER) admission during the 6-month follow-up following discharge from the index hospitalization. Post-discharge healthcare utilization in the 6-month follow-up was also characterized in the abstinent and non-abstinent cohorts using Berenson-Eggers Types of Service (BETOS) categories [11], which includes evaluation and management, procedures, imaging, tests, durable medical equipment, others and unclassified. Propensity score matching and 
other analyses were performed on Stata Software (version 15.1, Statacorp).

\section{Results}

Of the 600 patients enrolled in the clinical trial, 144 could be contacted and self-reported 7 day point prevalence abstinence at 6 months after hospital discharge. For 99 of these patients, it was possible to find an acceptable match among trial patients who could be contacted and reported current smoking. Even with this selection, propensity score matching could not completely balance the two cohorts in terms of their clinical indication. Therefore, this was included as a simple covariate in the cost regressions. Pre- and post- matching balance is given in Additional file 1 . The mean age of those included in this analysis was 47.5 years. Post-matching $p$-values showed no statistical difference in covariates and thus good balance. Other variables not included in the propensity matching, including marital status, insurance status, receipt of surgery during admission, and comorbidity counts were compared between abstainers and non-abstainers and no significant differences were found (Table 1). Therefore, 6 month follow-up cost comparisons were performed on unadjusted costs.

The cost of index hospitalization were significantly greater in patients who abstained compared to those that did not abstain (mean difference in costs of $\$ 3042$, higher for abstainers, 95\% CI $\$ 170$ to $\$ 5913, P=0.038$ ) (Table 2). However, the 6-month follow-up costs of abstainers and non-abstainers were not different, with large confidence intervals for the estimated differences ( $\$ 4196$ higher in abstainers, $95 \%$ CI $-\$ 6301$ to $\$ 14,693, p=0.433$ ) (Table 2). Adjusting the 6-month costs for the cost of the index hospitalization had little effect on this finding. There was no significant difference in the number of inpatient hospitalizations or emergency room visits between abstainers and non-abstainers in the 6 months after hospital discharge (Table 3). When utilization categories of follow-up were broken down further into categories such as imaging, evaluation and management, and procedures, there were also no differences in any category between abstainers and non-abstainers (Table 4). A full listing of diagnostic categories for the index hospitalization of abstainers vs. nonabstainers is presented in Additional file 2. When sorted by type of diagnostic category, the mean cost of initial hospitalization for abstainers was higher in 9 of 14 categories and median cost for abstainers was higher in 7 of 14 categories compared to non-abstainers (Additional file 3). A similar pattern of results was observed for the 6-month follow-up costs.

\section{Discussion}

The major finding of this study is that in a geographicallybased cohort of cigarette smokers admitted to a single

Table 1 Subject demographics

\begin{tabular}{lll}
\hline & Abstainers $^{\mathrm{a}}(\mathrm{N}=99)$ & Non-abstainers $(\mathrm{N}=99)$ \\
\hline Marital status & & $25(25.3 \%)$ \\
Divorces & $25(25.3 \%)$ & $29(29.3 \%)$ \\
Married & $30(30.3 \%)$ & $0(0.0 \%)$ \\
Separated & $4(4.0 \%)$ & $38(38.4 \%)$ \\
Single & $37(37.4 \%)$ & $7(7.1 \%)$ \\
Widowed & $3(3.0 \%)$ & \\
Insurance status & & $63(63.6 \%)$ \\
Government & $52(52.5 \%)$ & $4(4.0 \%)$ \\
No insurance & $5(5.1 \%)$ & $32(32.3 \%)$ \\
Private & $38(38.4 \%)$ & $0(0.0 \%)$ \\
Self pay & $4(4.0 \%)$ & $66(66.7 \%)$ \\
Surgery during hospitalization & & $33(33.3 \%)$ \\
No & $63(63.6 \%)$ & \\
Yes & $36(36.4 \%)$ & $2.8(2.0)$ \\
Count of Elixhauser comorbidities & & 3.0 \\
Mean (SD) & $2.7(1.9)$ & $1.0,4.0$ \\
Median & 2.0 & $0.0-11.0)$ \\
Q1, Q3 & $1.0,4.0$ & 0.65 \\
Range & $(0.0-9.0)$ & 0.90 \\
\hline
\end{tabular}

${ }^{\text {a }}$ Self-reported abstinence at 6 months after hospital discharge 
Table 2 Index hospitalization and 6-month follow-up costs of propensity -matched abstainers and non-abstainers

\begin{tabular}{|c|c|c|c|}
\hline & Mean & $95 \% \mathrm{Cl}$ & $p$-value \\
\hline \multicolumn{4}{|c|}{ Index hospitalization costs } \\
\hline Non-abstainers & $\$ 12,471.74$ & $\$ 10,818.27$ to $\$ 14,125.22$ & \\
\hline Abstainers $^{\mathrm{a}}$ & $\$ 15,513.28$ & $\$ 13,091.74$ to $\$ 17,934.83$ & \\
\hline Difference & $\$ 3041.54$ & $\$ 170.13$ to $\$ 5912.95$ & 0.038 \\
\hline \multicolumn{4}{|c|}{ 6-month follow-up costs } \\
\hline Non-abstainers & $\$ 18,789.63$ & $\$ 13,185.05$ to $\$ 24,394.21$ & \\
\hline Abstainers $^{a}$ & $\$ 22,985.64$ & $\$ 11,004.71$ to $\$ 34,966.57$ & \\
\hline Difference & $\$ 4196.01$ & $-\$ 6300.65$ to $\$ 14,692.68$ & 0.433 \\
\hline \multicolumn{4}{|c|}{ 6-month follow-up costs, adjusted for index hospitalization costs } \\
\hline Non-abstainers & $\$ 18,759.77$ & $\$ 13,187.24$ to $\$ 24,332.29$ & \\
\hline Abstainers $^{\mathrm{a}}$ & $\$ 23,007.87$ & $\$ 10,943.85$ to $\$ 35,071.89$ & \\
\hline Difference & $\$ 4248.10$ & $-\$ 6332.37$ to $\$ 14,828.58$ & 0.431 \\
\hline
\end{tabular}

Follow-up costs are cumulative since the date of hospital discharge

${ }^{a}$ Self-reported abstinence at 6 months after hospital discharge

institution, there was no evidence of an association between self-reported abstinence and health care costs or utilization over the first 6 months after hospital discharge.

Most studies of the incremental healthcare costs associated with smoking utilize models that include the estimated costs of smoking-related diseases and how smoking affects their prevalence. Several studies have used this method to estimate the economic benefits of quitting smoking in patients with medical conditions. Studies of inpatient smoking cessation interventions using this approach suggest significant cost benefits of interventions starting as early as 3 months postintervention, and virtually all show cost effectiveness by 1 year [12-14]. Similar studies that use this method to estimate the economic benefits of quitting for specific smoking-related diseases, such as myocardial infarction or stroke, also show significant medical cost benefits to quitting smoking starting about 1 year after cessation, which are sustained throughout the study period (several years, in some cases) $[12,13,15]$. However, few studies have attempted to actually measure changes healthcare costs associated with quitting in individual patients. Studies examining the economic impact of quitting

Table 3 Inpatient readmission and Emergency Room (ER) utilization by abstainers and non-abstainers

\begin{tabular}{lllc}
\hline & Non-abstainers & Abstainers $^{\mathrm{a}}$ & $p$-value \\
\hline No admission & $67(67.7 \%)$ & $61(62.9 \%)$ & 0.48 \\
Admission & $32(32.3 \%)$ & $36(37.1 \%)$ & \\
No ER Visit & $42(42.4 \%)$ & $35(36.1 \%)$ & 0.36 \\
ER Visit & $57(57.6 \%)$ & $62(63.9 \%)$ & \\
\hline
\end{tabular}

Values are $\mathrm{n}(\%)$

${ }^{\text {a }}$ Self-reported abstinence at 6 months after hospital discharge
Table 4 Utilization in the Berenson-Eggers Types of Service (BETOS) categories

\begin{tabular}{|c|c|c|c|c|c|c|}
\hline & $\mathrm{n}$ & Mean & SD & Median & IQR & Min/Max \\
\hline \multicolumn{7}{|c|}{ Evaluation \& management } \\
\hline Non-abstainers & 99 & 24.0 & 31.9 & 13 & 6,27 & $1 / 203$ \\
\hline Abstainers $^{\mathrm{a}}$ & 94 & 20.2 & 21.9 & 12.5 & 4,29 & $1 / 94$ \\
\hline \multicolumn{7}{|c|}{ Durable medical equipment } \\
\hline Non-abstainers & 35 & 4.5 & 6.2 & 2 & 1,6 & $1 / 29$ \\
\hline Abstainers $^{\mathrm{a}}$ & 26 & 2.8 & 2.9 & 1 & 1,4 & $1 / 12$ \\
\hline \multicolumn{7}{|l|}{ Imaging } \\
\hline Non-abstainers ${ }^{\mathrm{a}}$ & 82 & 7.9 & 10.3 & 5 & 3,8 & $1 / 67$ \\
\hline Abstainers $^{\mathrm{a}}$ & 82 & 7.0 & 10.2 & 4 & $2 / 8$ & $1 / 77$ \\
\hline \multicolumn{7}{|l|}{ Procedures } \\
\hline Non-abstainers ${ }^{\mathrm{a}}$ & 75 & 14.0 & 23.0 & 4 & 3,13 & $1 / 109$ \\
\hline Abstainers $^{\mathrm{a}}$ & 72 & 9.6 & 13.6 & 5 & $2,10.5$ & $1 / 79$ \\
\hline \multicolumn{7}{|l|}{ Tests } \\
\hline Non-abstainers & 93 & 15.8 & 20.2 & 8 & 4,20 & $1 / 111$ \\
\hline Abstainers $^{a}$ & 91 & 13.3 & 17.4 & 7 & 3,18 & $1 / 93$ \\
\hline \multicolumn{7}{|l|}{ Unclassified } \\
\hline Non-abstainers & 62 & 5.2 & 6.8 & 3 & 1,6 & $1 / 39$ \\
\hline Abstainers $^{\mathrm{a}}$ & 56 & 6.6 & 10.3 & 3 & 2,7 & $1 / 54$ \\
\hline \multicolumn{7}{|l|}{ Other } \\
\hline Non-abstainers & 83 & 11.6 & 17.7 & 6 & 2,11 & $1 / 107$ \\
\hline Abstainers ${ }^{a}$ & 80 & 9.3 & 12.6 & 5 & 2,12 & $1 / 81$ \\
\hline
\end{tabular}

${ }^{\text {a }}$ Self-reported abstinence at 6 months after hospital discharge $S D$ Standard deviation, IQR Interquartile range, Min minimum value, Max Maximum value

ideally would randomize patients to absolutely quit smoking or continue smoking, which would be neither feasible nor ethical. Thus, such studies typically utilize observational designs, which have the potential for confounding. For example, some studies find that quitting in ambulatory populations is actually associated with an increase in annual health care costs, which may last for several years before declining [16-19]. The most likely explanation for this seeming paradox is the "teachable moments" effect of illness and hospitalization; those patients who are more ill are more likely to quit. Thus, such increases in healthcare costs may reflect the severity of the underlying illness that prompted quitting, rather than an effect of abstinence itself. Also, although abstinence from cigarettes has immediate physiologic benefits, it may take some time before there are significant improvements (or arrest in the rate of decline) in smoking-related diseases such as chronic obstructive pulmonary disease that could translate to a reduction in costs.

When considering the potential impact of quitting on actual costs, it is also important to distinguish between study designs that compare costs according to smoking 
status (typically as current, former, and never smokers) and those that assess costs in smokers who do and do not quit. Regarding the former design as applied to hospitalized smokers, a prior analysis that also included smokers who were enrolled in the present clinical trial found no association between smoking status and hospital costs for either medical or surgical patients [20]. In another analysis restricted to surgical patients, costs in the first year after hospitalization were higher in current smokers compared with never smokers; medical patients were not included in this analysis [5]. Another study found a small but significant increase in the cost of hospitalization in surgical patients who were current smokers compared to those who were never or former smokers; post hospitalization costs were not studied [21]. These are interesting analyses, but may not be useful to infer the potential effect of quitting on costs and utilization; costs may well differ in recent quitters compared with never smokers.

To our knowledge, no prior study has compared post hospitalization costs and utilization in patients who did and did not quit smoking after hospitalization, so there are no data directly comparable to our results. The original intent at the time that the randomized trial was planned was to analyze post hospitalization costs according to treatment assignment, hypothesizing that postoperative abstinence rates would differ between groups, and that the economic impact of these differential rates could be analyzed. However, there was no difference in quit rates according to treatment assignment, thus no rationale to perform this originallyplanned economic analysis. Nonetheless, these data provided the opportunity to examine the association between quitting and post hospitalization costs, albeit with the same potential limitations inherent in other observational studies. In particular, it is possible that differences in patient characteristics not accounted for in the analysis, such as severity of illness, contribute to the lack of difference in costs between those who did and did not quit. More severe illness may contribute to both the likelihood of quitting and increased costs, obscuring any potential benefit of abstinence on healthcare utilization and costs. This possibility is supported by the finding that the costs of the index hospitalization of the patients that abstained after discharge were higher, as both groups of patients were abstinent while in hospital. It is also possible that differences might have become apparent if subjects were followed for a longer period of time. Thus, these results cannot be used to evaluate the potential economic benefit of a smoking cessation intervention. Nonetheless, we find no evidence that quitting is associated with a decrease in initial post-hospitalization costs. Thus, although there are certainly many good reasons to treat hospitalized patients for tobacco dependence, there is not yet evidence that such treatment is associated with immediate economic benefit. Rather, based on the results of long-term longitudinal studies of costs showing that economic benefits take some time to accrue, it may also take a considerable period of time before similar benefits would be observed in hospitalized patients.

Although there are several strengths of our study design, including the fact that participants were drawn from an area of geographic residence (reducing the potential for referral bias) and a billing resource that allowed for complete ascertainment of costs using a validated methodology, this study has some significant limitations. First, the number of patients available for analysis was relatively small, and, as typical of cost studies, there was large variation in costs among cases. Some of those who abstained were not included in the analysis because a suitable matched patient could not be identified. This limits the ability to detect differences in costs. Second, as with any observational study, there is the potential for confounding factors not captured in our analytic techniques employed to adjust between groups (such as severity of illness, as discussed). The analysis relied on self-reported smoking status at 6 months, as only about half of the patients in the clinical trial provided urine samples for biochemical confirmation [6]. Although the results among those who provided samples was reassuring regarding the general accuracy of self-report, it is possible that not all selfreported abstainers actually were abstaining, which would bias against finding differences in costs if they existed. We ascertained 7-day point prevalence abstinence rather than continuous abstinence, and it is possible that some patients who were abstinent at 6 months had smoked at some time between hospital discharge and this time. However, there is a good correlation between point prevalence and continuous abstinence, and both methods produce similar results when applied to clinical trials [22]. Finally, this analysis includes a single hospital; a multi-center design would be desirable for future studies.

In conclusion, this study found no evidence to support the hypothesis that maintaining abstinence after hospital discharge is associated with a decrease in health care costs or utilization over the first 6 months after hospital discharge. These results may suggest caution in using the incremental costs of smoking-related illnesses to estimate the immediate potential economic impact of smoking cessation in medical populations. However, because this study had several limitations, including relatively small size and relatively large interindividual differences in in health care costs, larger studies will be needed to more adequately address this important question. 


\section{Supplementary information}

Supplementary information accompanies this paper at https://doi.org/10. 1186/s12913-019-4777-7.

Additional file 1. Pre- and post-matching balance of age, sex and clinical indication propensity score matching of abstainers and nonabstainers.

Additional file 2. Frequency of diagnoses by 6-month abstinence.

Additional file 3. Mean and median costs of index hospitalization and 6-month follow-up costs by indication for subjects who continued to smoke versus those who were abstinent at 6-month follow-up, according to diagnostic category.

\section{Abbreviations}

BETOS: Berenson-Eggers Types of Service; CCS: Clinical Classification Software; $\mathrm{Cl}$ : Confidence intervals; ER: Emergency room; GLM: Generalized linear modeling; MN: Minnesota

\section{Acknowledgements}

Not applicable.

\section{Authors' contributions}

MBN helped conceptualize the manuscript, manage, analyze and interpret the data, and drafted the manuscript. BJB helped conceptualize the manuscript, led efforts to analyze and interpret the data, and assisted in drafting the manuscript. JPM took the lead in performing the actual data analysis, and drafted significant portions of the manuscript. DOW conceived and designed the study, obtained funding, conceptualized the manuscript, interpreted the data, and oversaw the preparation of the manuscript. All authors read and approved the final manuscript.

\section{Funding}

The underlying study was funded by grant RC-2012-0001 from Clearway Minnesota. The study sponsor had no role in the study design, collection, analysis, or interpretation of the data, the writing of the report, or the decision to submit the report for publication.

\section{Availability of data and materials}

This dataset regarding patient costs is proprietary information of the Mayo Clinic and Olmsted Medical Center, and is not publically available.

\section{Ethics approval and consent to participate}

This study was approved by the Mayo Clinic and Olmsted Medical Center Institutional Review Boards, Rochester, MN.

\section{Consent for publication}

Not applicable.

\section{Competing interests}

The authors declare that they have no competing interests.

\section{Author details}

'Department of Anesthesiology and Perioperative Medicine, Mayo Clinic, 200 First Street SW, Rochester, MN 55905, USA. ${ }^{2}$ Mayo Clinic Robert D. and Patricia E. Kern Center for the Science of Health Care Delivery, Mayo Clinic, Rochester, MN, USA.

Received: 1 October 2019 Accepted: 22 November 2019 Published online: 02 December 2019

\section{References}

1. Orleans $C T$, Kristeller JL, Gritz ER. Helping hospitalized smokers quit: new directions for treatment and research. J Consult Clin Psychol. 1993;61(5): 778-89.

2. Rigotti NA, Clair C, Munafo MR, Stead LF. Interventions for smoking cessation in hospitalised patients. Cochrane Database Syst Rev. 2012;5: CD001837.
3. Xu X, Bishop EE, Kennedy SM, Simpson SA, Pechacek TF. Annual healthcare spending attributable to cigarette smoking: an update. Am J Prev Med. 2015;48(3):326-33.

4. Lightwood J, Glantz SA. Smoking behavior and healthcare expenditure in the United States, 1992-2009: panel data estimates. PLoS Med. 2016;13(5): e1002020.

5. Warner DO, Borah BJ, Moriarty J, Schroeder DR, Shi Y, Shah ND. Smoking status and health care costs in the perioperative period: a population-based study. JAMA Surg. 2014;149(3):259-66.

6. Warner DO, Nolan MB, Kadimpati S, Burke MV, Hanson AC, Schroeder DR. Quitline tobacco interventions in hospitalized patients: a randomized trial. Am J Prev Med. 2016;51(4):473-84.

7. Clinical Classifications Software (CCS) for ICD-9 CM [available from https:// www.hcup-us.ahrq.gov/toolssoftware/ccs/ccs.jsp]. Accessed 27 Nov 2019.

8. Visscher SLNJ, Yawn BP, Reinalda MS, Anderson SS, Borah BJ. Developing a standardized healthcare cost data warehouse. BMC Health Serv Res. 2017; 17(1):396.

9. Using appropriate price indices for analyses of health care expenditures or income across multiple years (MEPS: Medical Expenditure Panel Survey) 2014. Available from https://meps.ahrq.gov/about_meps/Price_Index.shtml]. Accessed 27 Nov 2019.

10. Manning WG, Basu A, Mullahy J. Generalized modeling approaches to risk adjustment of skewed outcomes data. J Health Econ. 2005;24(3):465-88.

11. Berenson-Eggers Type of Service (BETOS) Codes [Available from https:// www.cms.gov/Research-Statistics-Data-and-Systems/Statistics-Trends-andReports/MedicareFeeforSvcPartsAB/downloads/betosdesccodes.pdf]. Accessed 27 Nov 2019.

12. Slatore CG, Au DH, Hollingworth W. Cost-effectiveness of a smoking cessation program implemented at the time of surgery for lung cancer. J Thorac Onc. 2009;4(4):499-504.

13. Meenan RT, Stevens VJ, Hornbrook MC, La Chance PA, Glasgow RE, Hollis JF, et al. Cost-effectiveness of a hospital-based smoking cessation intervention. Med Care. 1998:36(5):670-8.

14. Ekpu VU, Brown AK. The economic impact of smoking and of reducing smoking prevalence: review of evidence. Tob Use Insights. 2015;8:1-35.

15. Ladapo JA, Jaffer FA, Weinstein MC, Froelicher ES. Projected costeffectiveness of smoking cessation interventions in patients hospitalized with myocardial infarction. Arch Intern Med. 2011;171(1):39-45.

16. Hockenberry JM, Curry SJ, Fishman PA, Baker TB, Fraser DL, Cisler RA, et al. Healthcare costs around the time of smoking cessation. Am J Prev Med. 2012;42(6):596-601.

17. Fishman PA, Thompson EE, Merikle E, Curry SJ. Changes in health care costs before and after smoking cessation. Nicotine Tob Res. 2006;8(3):393-401.

18. Fishman PA, Khan ZM, Thompson EE, Curry SJ. Health care costs among smokers, former smokers, and never smokers in an HMO. Health Serv Res. 2003;38(2):733-49.

19. Suwa K, Yoshikawa R, Iwasaki K, Igarashi A. The association between smoking cessation outpatient visits and total medical costs: a retrospective, observational analysis of Japanese employee-based public health insurance data. J Med Econ. 2018;21(5):443-9.

20. Nolan MB, Moriarty JP, Warner DO. Economic analysis of a geographical cohort of hospitalized smoking patients. Mayo Clin Proc. 2018;93(8):1034-42.

21. Kamath AS, Vaughan Sarrazin M, Vander Weg MW, Cai X, Cullen J, Katz DA. Hospital costs associated with smoking in veterans undergoing general surgery. J Am Coll Surg. 2012;214(6):901-8 e1.

22. Hughes JR, Carpenter MJ, Naud S. Do point prevalence and prolonged abstinence measures produce similar results in smoking cessation studies? A systematic review. Nicotine Tob Res. 2010;12(7):756-62.

\section{Publisher's Note}

Springer Nature remains neutral with regard to jurisdictional claims in published maps and institutional affiliations. 